\title{
Noise Level and its Sources in Neonatal Intensive Care Units of Selected Public Hospitals in Kigali City
}

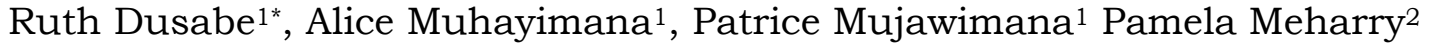 \\ ${ }^{1}$ School of Nursing and Midwifery, College of Medicine and Health Sciences, University of Rwanda, \\ Kigali, Rwanda \\ ${ }^{2}$ Department of Women's, Children's and Family Health Services, University of Ilinois, Chicago, \\ USA
}

*Corresponding author: Ruth Dusabe, School of Nursing and Midwifery, College of Medicine and Health Sciences, University of Rwanda, Remera Campus, Kigali City, Rwanda. Email:

milsruth@gmail.com.

\begin{abstract}
Background

Noise in the neonatal intensive care unit (NICU) has an impact on the developmental progress of neonates, especially preterm. Technology advancement has improved the life of preterm neonates, but also changed NICU into a noisy place.
\end{abstract}

\section{Objective}

To measure the noise levels and identify its sources in NICU at selected public hospitals in Kigali city

\section{Methods}

A quantitative cross-sectional descriptive study. A sound level meter was used in recording the Sound level in six different locations of the NICUs at five different times. An observational checklist was used to identify possible sources of noise. Data were analysed using descriptive and inferential statistics (ANOVA).

\section{Results}

Sound levels recorded in all the NICUs were high ranging from 61.8 decibel (dB) to $77.0 \mathrm{~dB}$, greater than the $45 \mathrm{~dB}$ recommended by the American Association of Pediatrics (AAP). The maximum noise level was $77.0 \mathrm{~dB}$. Ward rounds had the highest noise levels, and lunchtime had the lowest noise levels in all hospitals. Noise levels were high at the station near the entrance in all NICU rooms.

\section{Conclusion}

In all NICUs, the noise level generated was greater than the standard limits established by AAP. Neonates need noise-free environment as excess noise has an impact on their wellbeing.

Rwanda J Med Health Sci 2020;3(2):115-127

Keywords: Neonatal intensive care unit, NICU, noise level, neonates, sound

\section{BACKGROUND}

Despite the WHO and AAP recommendations, existing analyses of the acoustic environment in the NICU have indicated that noise standards are being exceeded regularly. Studies done in different NICU environments have demonstrated that average noise levels range from 48 to $55 \mathrm{~dB}$.[1] Another study found that sound levels exceeded the recommended standard, and the noise level was 84 dB.[2] Noise level in NICU is produced by various sources, which may exert undesirable physiological effects on the neonates.[3] The noise level in NICU is extremely diverse; heated cribs, incubators, mechanical ventilators, monitor alarms, infusion pumps, opening and closing of doors and draws, circulation 
of people and conversations.[4]

A WHO revealed that noise exposure caused global disability and estimated that in Western Europe 45,000 Disability Adjusted Life Years (DALYs) are lost for noise-induced cognitive impairment of children, 61,000 DALYs for cardiovascular diseases and 903,000 DALYs for sleep disturbance.[5] A study conducted by Aly and Ahmed found that the vital signs of preterm were affected by noise more than full-term neonates.[4] Preterm neonates are more affected by noise and may spend more time hospitalized in the NICU needing special care.

NICU standard noise limits were developed a long time ago and have not changed. In 1974, the Environmental Protection Agency (EPA) set an average sound level limit of $45 \mathrm{~dB}$ for hospitals based on the best evidence at the time.[6] In 1997, the AAP applied this recommendation for sound levels not to exceed $45 \mathrm{~dB}$ in NICUs.[6] Since that time, significant advancement in NICU medical technology has impacted the life of neonates, including the high and problematic incidence of neurodevelopmental disorders and disability among survivors.[6]

Worldwide every year, 15 million neonates are born preterm, and those that survive may suffer from disability throughout their lives, especially related to auditory problems, visual and learning difficulties, [7] mostly caused by high noise in NICU. More than one million die because of complications. [8] In Rwanda, 35,000 neonates are born preterm each year, and 2,600 neonates die due to preterm complications they may face during hospitalization. [8] Disabling hearing loss in children was found in 34 million $(7.3 \%)$ of 466 million people, and the highest numbers were in South Asia, the Asia Pacific, and sub-Saharan Africa.[9]

According to Schokry, preterm neonates exposed to repeated loud noise in the NICU are more at risk of having sensory neural problems with hearing loss and developmental delay.[10] Exposing preterm neonates to noise will have a significant impact on their lives, as most of their bodies are not yet well developed. This early exposure to noise has the potential effect on preterm neonatal auditory development, sleep patterns, and physiological stability, thus impacting developmental progress.[5] The noise level measurement in a neonatal unit is necessary to compare to what is recommended. This location is very different from the protective intrauterine environment where the neonate was living. The neonate comes out of a calm, quiet, dark, and cushioned environment, to an environment with excessive light and noise, a constant movement of people and interruptions of sleep and wakefulness, often with discomfort and pain.[2]

The physiological effects of excessive noise include changes in heart rhythm, increased blood pressure, peripheral vasoconstriction, and dilation of the pupils with increased secretions of adrenaline.[11] These changes affect the physiological and neurobehavioral states of the neonate.[12] High noise also causes disturbances in sleep patterns, agitation, and irritability, crying, increased oxygen consumption, fatigue and increased heart rate.[2] A study done by Almadhoob and Ohlsson demonstrated that $52 \%$ of preterm neonates treated in the NICU had abnormal audiograms due to noise leading to impaired hearing.[13] Improved technology for caring for preterm infants has been accompanied by concerns about the impact of NICU noise to these neonates.[10] It is important to identify and measure the noise level in the NICU in order to protect neonates from a harmful environment. This study will contribute to the existing knowledge on determining noise levels in different NICUs in Rwanda.

\section{METHODS}

\section{Design}

A non-experimental simple descriptive crosssectional study design was conducted in NICUs of selected hospitals in Kigali city to assess the noise level and possible sources of noise. The layout of the NICU is illustrated in Figures 1-4 showing the location where sound levels were taken. The study was conducted over one month from March 18 to April 7, 2019. 


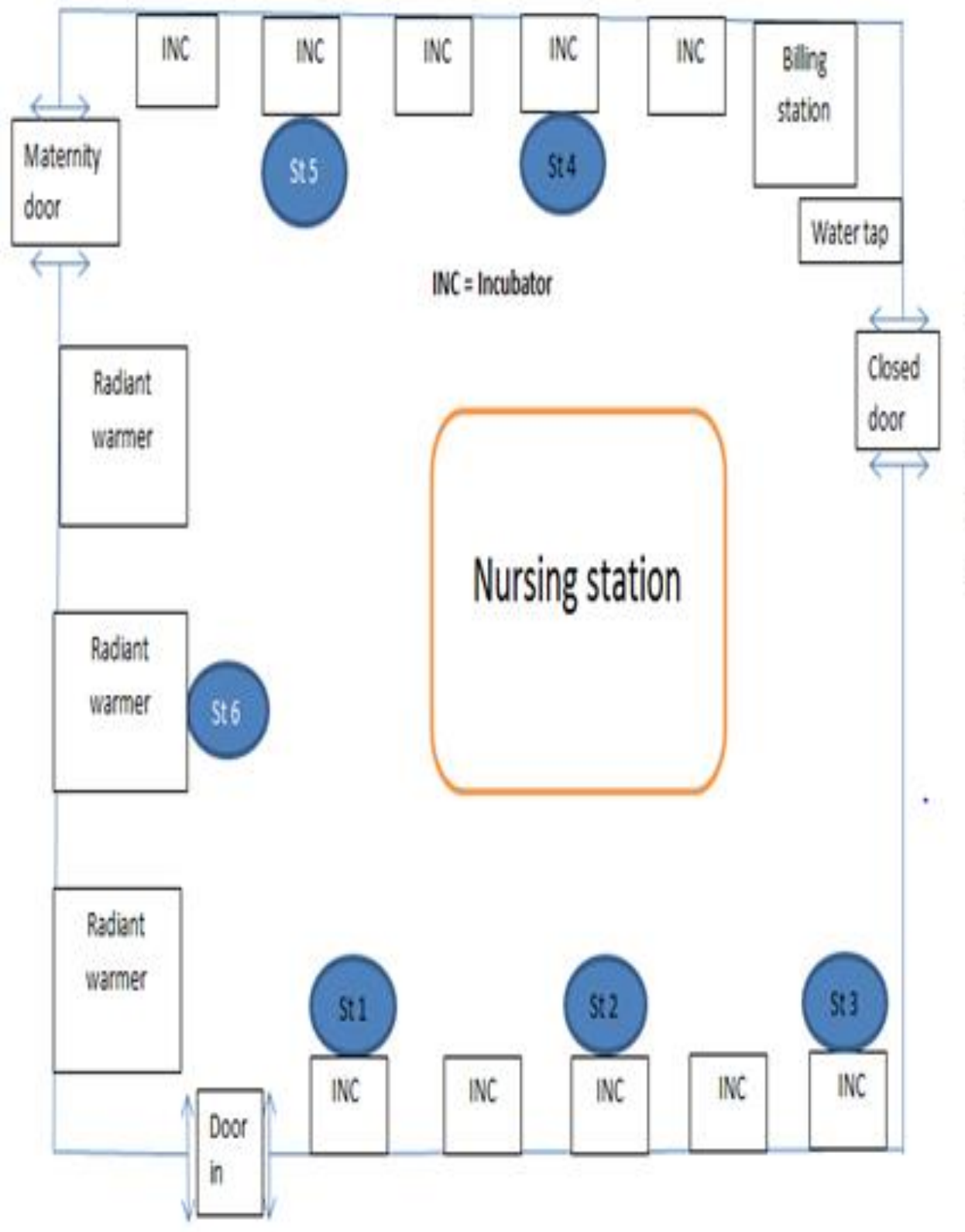

Station l: nearest the entrance and near nursing station

Station 2: nearest the nursing station

Station 3: further from nursing station

Station 4: nearest the nursing station

Station 5: furthest from the nursing station

Station 6: near the nurse station

Figure 1. Hospital 1: Referral hospital 


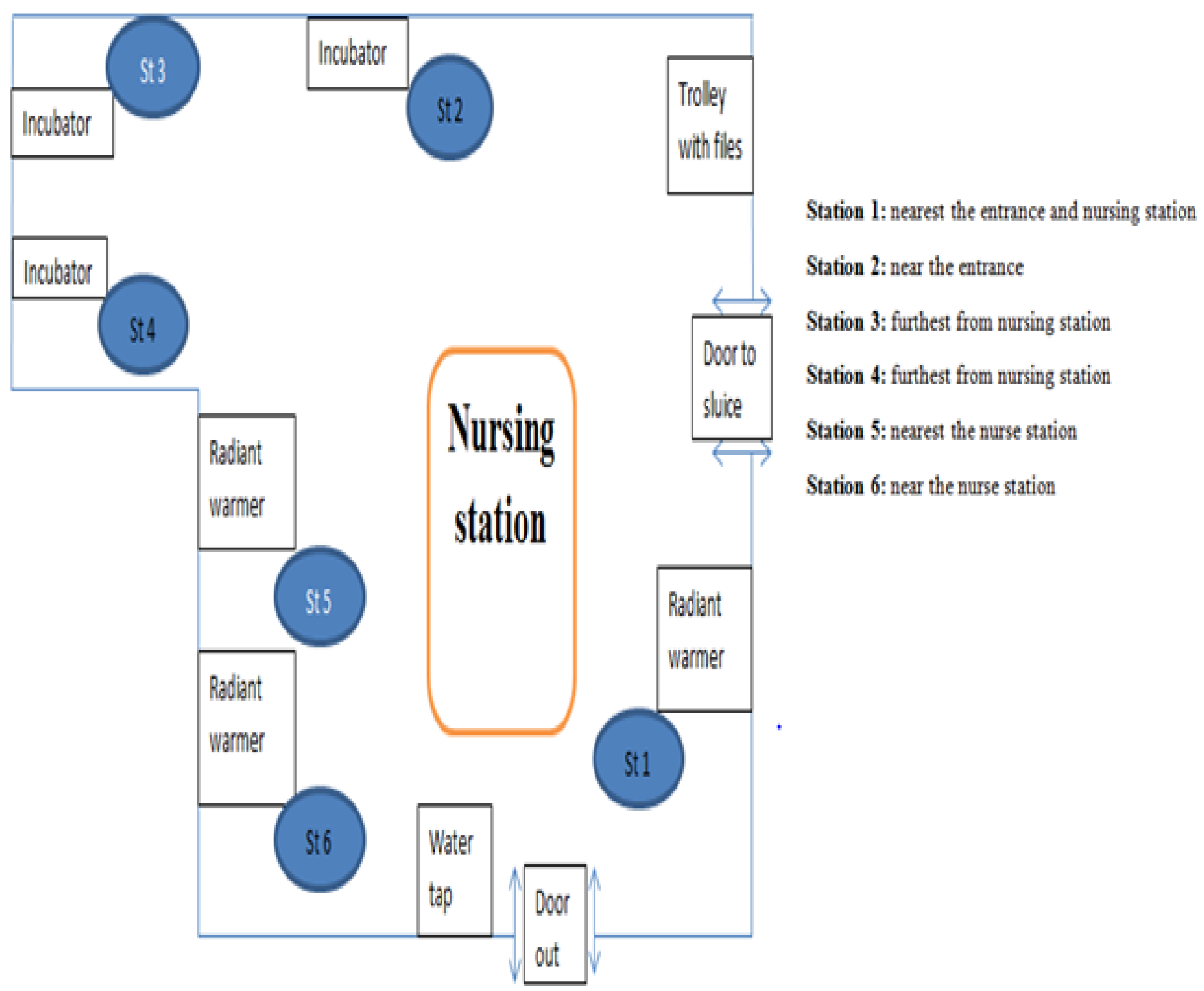

Figure 2. Hospital 2: Referral hospital 


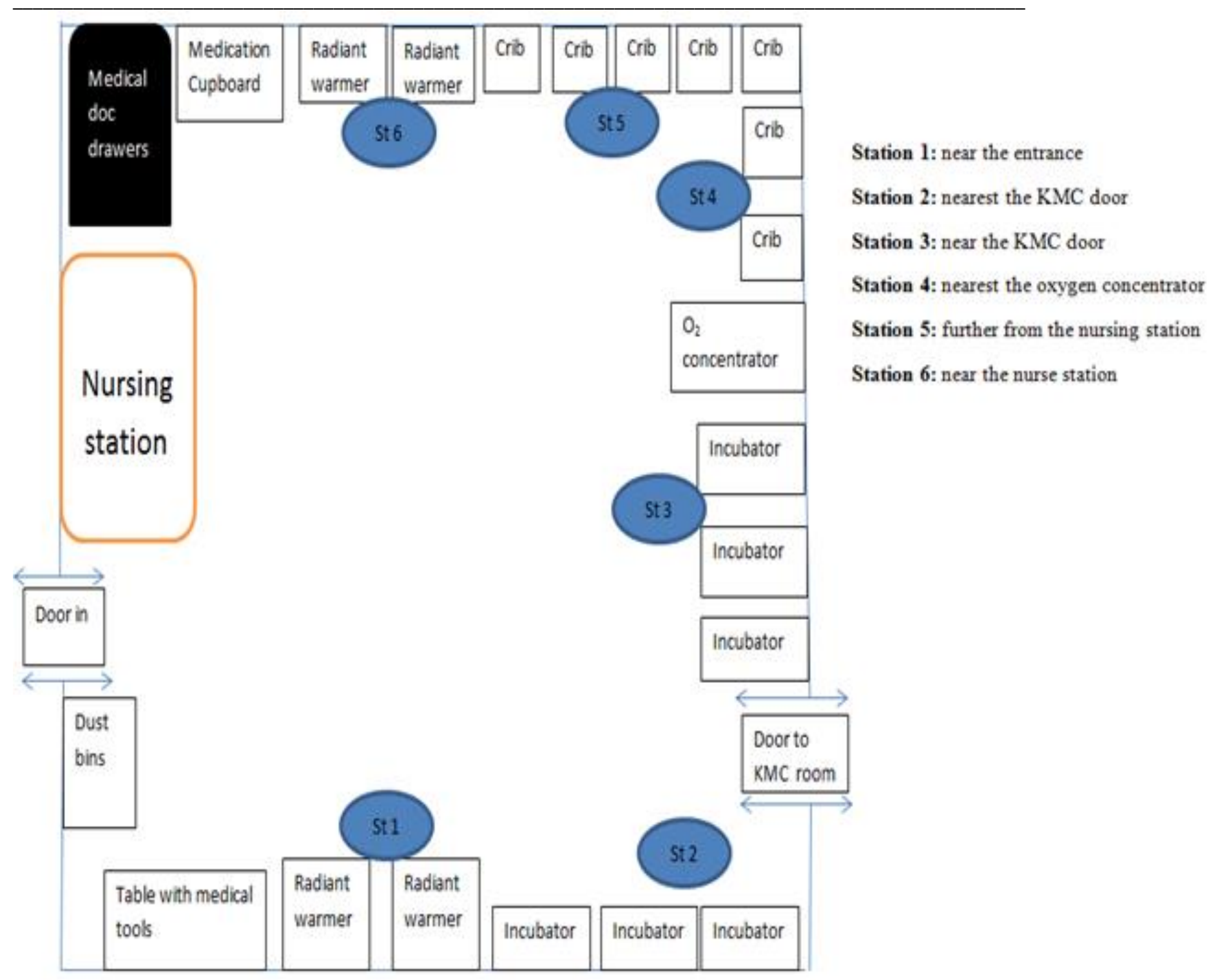

Figure 3. Hospital 3: District hospital 


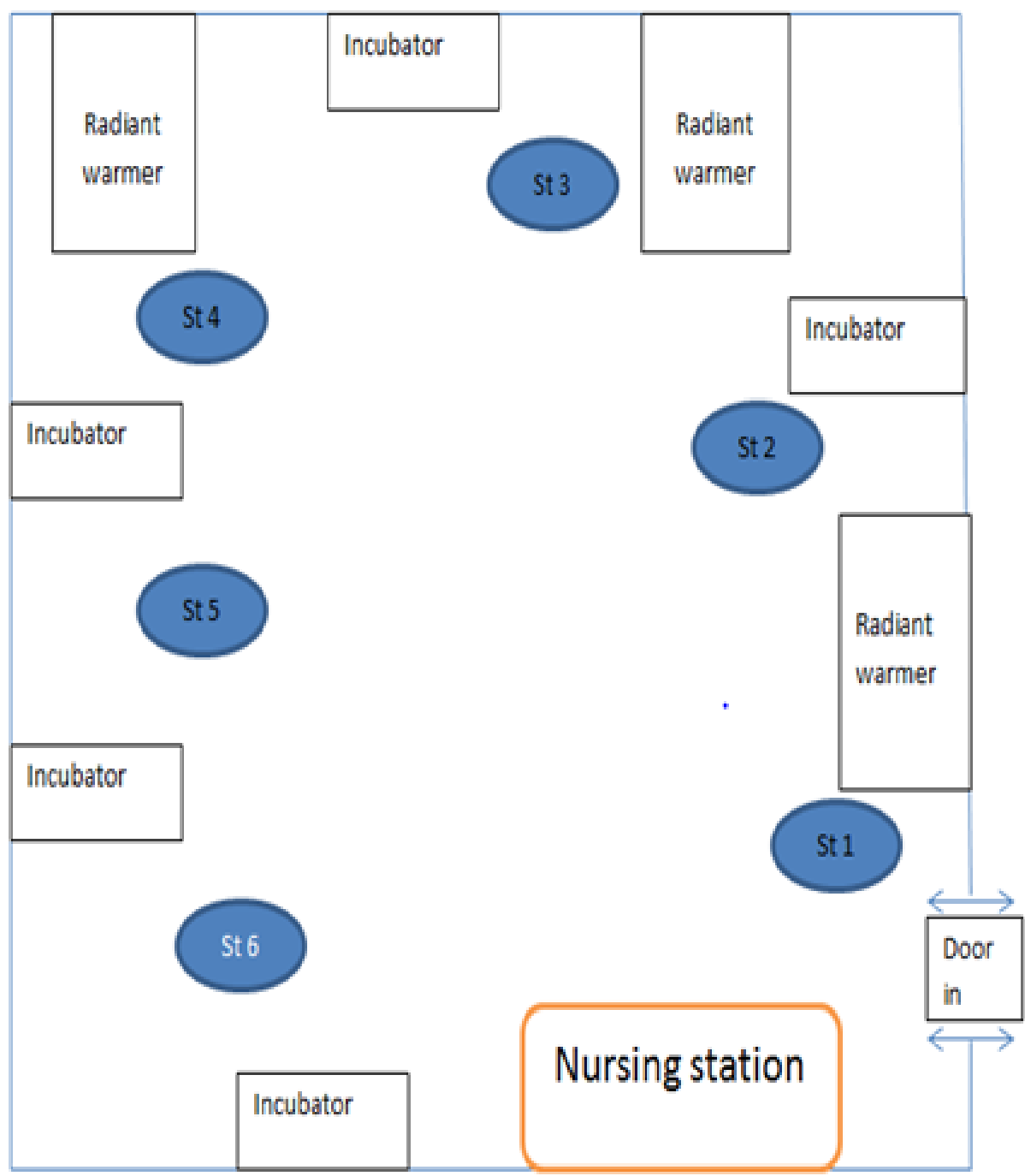

tion 1 : nearest the entrance and near nursing station

tion 2: further from the nursing station

tion 3: further from nursing station

tion 4 : furthest from the nursing station

tion 5: furthest from the nursing station

Figure 4. Hospital 4: District hospital

\section{Participants' recruitment}

Simple random sampling was used to select hospitals. A sampling strategy was used to get time intervals, where data were collected over different times of the day over seven days. This time was collected purposively where one hourly interval was recorded according to different shifts of the day; morning time $(7: 00$ am to $8: 00 \mathrm{am})$, ward round with midwives/nurses students, resident doctors, pediatric doctors, and midwives/ nurses. Lunchtime (12:00 pm to 1:00 pm) when HCP has gone, only one or two remains, midnight (12:00 am to $1: 00 \mathrm{am})$ all these were applied to all hospitals except during shift-changing where two hospitals were done at 5:00 pm to $6 \mathrm{pm}$, and other two were done at $7: 00 \mathrm{pm}$ to $8: 00 \mathrm{pm}$. Five timeframe multiplied by six stations $\left(5^{*} 6\right)$ generated $=30$ samples in a single day. Noise levels were measured in seven days in each NICU among four different settings. Total sample per hospital in a week $=30 * 7=210$. Total sample from all four hospitals $=210 * 4=840$. Total sound levels were 840 records.

\section{Measures}

Sound level readings were taken with a Sound level meter called Velleman DEM 200 instrument which measures noise between $30 \mathrm{~dB}$ to $130 \mathrm{~dB}$, resolution $0.1 \mathrm{~dB}$, accuracy: +/-1.4 $\mathrm{dB}$, frequency weighting: A; approximates function characteristics of the human ear. An observation checklist was used for possible sources of noise given by $\mathrm{J}$ Neille, which was used in their study.[5] 
Section 1: Sound levels on different days (Monday, Tuesday, Wednesday, Thursday, Friday, Saturday, and Sunday) at the four hospitals were measured in $\mathrm{dB}$. Means, minimum and maximum sound levels, confidence interval, and p-value were used (Table 2).

Section 2: Sound levels in different shifts through the days; these were done at four hospitals and were measured in $\mathrm{dB}$. Mean, minimum, and maximum sound levels, confidence interval, and p-value were used (Table 3).

Section 3: Sound levels were compared with the AAP recommendation. Different days of sound levels and different shifts were measured in $\mathrm{dB}$ (Figure 5).

\section{Data collection}

The sound level in NICU was measured using a sound level meter device. The meter was calibrated before starting measurements at each new measurement location. Readings were taken in six different areas/stations at five different times of the day (morning, ward round, lunchtime, shift-change, and midnight). The same stations were also used for the whole week of data collection. The sound level within each NICU was identified by means of taking six readings (from stations) at each time, and the readings were then averaged to get sound level at each time of the day. The noise level was corrected for the whole week at each hospital. A data capture sheet was used in the documentation of data collected. The observational checklist was also used to assess the possible sources of noise.

Two research assistants were midwifery staff. One was working during the day up to shift-changing, and the other was taking midnight measurements. They were used because data were collected from morning to midnight during the different time interval for the whole week, at each hospital. They were trained on the use of the instrument and how to use the observational checklist. The investigator was the one to supervise them daily, and the report was given.

\section{Data analysis}

Data collected were analyzed using descriptive statistics in terms of mean, standard deviation, maximum, and minimum, which were presented in figures and tables. Inferential statistics were also used, where one-way analysis of variance (ANOVA) was used to assess the significance and direction of the relationship between the time of the day, station, different days, and the level of noise within each hospital. Data were entered in Microsoft's Excel 13 and then imported in the statistical package for Social Sciences (SPSS) version 22 which was used in data analysis. Maximal and minimal values were also calculated for each time in each station along with their standard deviation (SD) to summarize the data. All results were reported as $\mathrm{dB}$ (logarithmic scale), the mean of sound levels as compared to the current $45 \mathrm{~dB}$ recommendation by Statistical significance was set at $\mathrm{p}<0.05$.

\section{Ethical considerations}

Approval was obtained from the Institutional Review Board (IRB) of the University of Rwanda College Medicine and Health Sciences. Directors General of the four selected hospitals permitted the investigator to conduct the study.

\section{RESULTS}

Findings from this study determined the noise levels and its sources in the neonatal care units (NCU) of four public hospitals (two districts and two referrals) in Kigali City. 
Table 1. Summary of important descriptive characteristics of each hospital

\section{HOSPITALS}

\begin{tabular}{|c|c|c|c|c|}
\hline & $\mathbf{1}$ & 2 & 3 & 4 \\
\hline \multirow[t]{2}{*}{ Number of beds } & 10 Inc $\&$ & 3 Inc $\&$ & 5 Inc, & 5 Inc $\&$ \\
\hline & $\begin{array}{l}3 \mathrm{RW}=13 \text { in an } \\
\text { open room }\end{array}$ & $\begin{array}{l}3 \mathrm{RW}=6 \text { but } \\
\text { may increase } \\
\text { according to the } \\
\text { need. All in an } \\
\text { open room }\end{array}$ & $\begin{array}{l}4 \mathrm{RW} \& \\
7 \mathrm{cribs}=16 \mathrm{in} \\
\text { an open room }\end{array}$ & $\begin{array}{l}3 \mathrm{RW}=8 \text { in an } \\
\text { open room }\end{array}$ \\
\hline \multirow[t]{2}{*}{ Number of HCP } & *5 day duty & *5 day duty & *5 day duty & *4 day duty \\
\hline & *3 night duty & *4 night duty & *2 night duty & *2 night duty \\
\hline $\begin{array}{l}\text { Number of neonates } \\
\text { admitted in NICU }\end{array}$ & 12 & 6 & 16 & 8 \\
\hline $\begin{array}{l}\text { Feeding time and } \\
\text { personnel to feed }\end{array}$ & $\begin{array}{l}\text { Every } 3 \text { hours. } \\
\text { Mother is } \\
\text { responsible }\end{array}$ & $\begin{array}{l}\text { Every } 3 \text { hours. } \\
\text { Nurses are } \\
\text { responsible }\end{array}$ & $\begin{array}{l}\text { Every } 3 \text { hours. } \\
\text { Mother is } \\
\text { responsible }\end{array}$ & $\begin{array}{l}\text { Every } 3 \text { hours. } \\
\text { Mother is } \\
\text { responsible }\end{array}$ \\
\hline Loudest station & $\begin{array}{l}\text { Near the entrance } \\
\text { on station } 1 \\
(63.7 \mathrm{~dB})\end{array}$ & $\begin{array}{l}\text { Near the } \\
\text { entrance on } \\
\text { station } 1 \\
(64.8 \mathrm{~dB})\end{array}$ & $\begin{array}{l}\text { Near the } \\
\text { entrance on } \\
\text { station } 1 \\
(67.1 \mathrm{~dB})\end{array}$ & $\begin{array}{l}\text { Near the } \\
\text { entrance on } \\
\text { station } 1 \\
(65.5 \mathrm{~dB})\end{array}$ \\
\hline
\end{tabular}

RW: Radiant Warmer Inc: Incubator

Table 1 above is the summary of an important description of the study. It demonstrates the characteristic of each NICU; the number of beds, number of HCP per shift, number of neonates admitted, and the loudest station.

Table 2. Sound levels on different days at all hospitals

\begin{tabular}{llllllll}
\hline Hospital & Days & $\begin{array}{l}\text { Mean } \\
(\mathbf{d B})\end{array}$ & SD & Min & Max & $\mathbf{9 5 \%}$ CI & $\begin{array}{l}\text { p- } \\
\text { Value }\end{array}$ \\
\hline \multirow{4}{*}{1} & Monday & 62.0 & 1.4 & 58.9 & 65.8 & $61.5-62.5$ & \\
& Tuesday & 61.8 & 1.6 & 57.3 & 63.5 & $61.2-62.4$ & 0.004 \\
& Wednesday & 63.4 & 2.7 & 60.3 & 72.3 & $62.2-64.3$ & \\
& Thursday & 62.5 & 2.6 & 56.8 & 69.5 & $61.4-63.4$ & \\
& Friday & 63.5 & 3.4 & 56.1 & 72.3 & $62.2-64.8$ & \\
& Saturday & 62.3 & 1.3 & 60.7 & 65.4 & $61.8-62.8$ & \\
& Sunday & 60.9 & 2.3 & 56.6 & 64.7 & $60.0-61.8$ & \\
& Monday & 64.2 & 2.3 & 58.0 & 70.5 & $63.3-65.0$ & \\
& Tuesday & 63.9 & 2.2 & 60.9 & 72.5 & $63.0-64.7$ & \\
& Wednesday & 64.7 & 2.1 & 61.3 & 70.0 & $64.0-65.4$ & \\
& Thursday & 65.6 & 2.1 & 61.6 & 69.6 & $64.8-66.4$ & 0.05 \\
& Friday & 63.9 & 2.3 & 58.0 & 68.2 & $63.1-64.8$ & \\
& Saturday & 64.0 & 2.3 & 59.9 & 69.1 & $63.2-64.9$ & \\
& Sunday & 64.4 & 2.4 & 59.7 & 71.9 & $64.0-65.2$ & \\
& Monday & 66.4 & 2.2 & 62.9 & 72.3 & $65.6-67.2$ &
\end{tabular}




\begin{tabular}{lllllllll}
\hline & Tuesday & 65.5 & 3.1 & 59.4 & 74.0 & $64.2-67.7$ & \\
Wednesday & 66.7 & 2.7 & 62.4 & 76.5 & $65.7-66.5$ & \\
Thursday & 66.1 & 1.7 & 63.0 & 71.3 & $65.4-66.7$ & \\
Friday & 67.7 & 1.9 & 64.0 & 72.7 & $66.9-68.4$ & 0.002 \\
Saturday & 65.6 & 1.7 & 63.0 & 70.3 & $64.9-66.3$ & \\
Sunday & 65.9 & 2.3 & 59.4 & 69.4 & $65.2-66.5$ & \\
Monday & 63.7 & 5.9 & 50.4 & 74.8 & $61.5-65.9$ & \\
Tuesday & 65.0 & 3.3 & 59.9 & 70.9 & $63.7-66.2$ & \\
& Wednesday & 63.0 & 5.1 & 54.9 & 74.0 & $61.1-64.9$ & \\
Thursday & 64.3 & 3.7 & 58.8 & 74.9 & $62.9-65.7$ & \\
& Friday & 65.2 & 3.7 & 58.5 & 72.2 & $63.8-66.5$ & 0.000 \\
& Saturday & 65.9 & 3.7 & 55.1 & 72.6 & $64.5-67.2$ & \\
& Sunday & 68.0 & 4.3 & 61.7 & 77.0 & $66.5-69.7$ & \\
& & & & & & & \\
& & & & & & &
\end{tabular}

SD: Standard Deviation Min: Minimum Max: Maximum CI: Confidence interval

Table 2 illustrated the results of hospitals $(1,2,3 \& 4)$ which were statistically significant $(p=0.004, p=0.05$, $\mathrm{p}=0.002, \mathrm{p}=0.000)$ respectively. The highest sound level was on Sunday at hospital $4(68.0 \mathrm{~dB})$ and the lowest was on Monday at hospital 1 (62.0 dB).

Table 3. Sound levels on different shifts at all hospitals

\begin{tabular}{llllllll}
\hline Hospital & Shift & $\begin{array}{l}\text { Mean } \\
(\mathbf{d B})\end{array}$ & SD & Min & Max & 95\% CI & $\begin{array}{c}\text { p- } \\
\text { Value }\end{array}$ \\
& & & & & & \\
& & & & & & & \\
& Morning & 62.7 & 2.1 & 57.2 & 66.8 & $62.1-63.3$ & \\
& Ward round & 63.9 & 3.1 & 57.7 & 72.2 & $62.9-64.9$ & \\
& Lunchtime & 62.0 & 1.7 & 56.8 & 65.3 & $61.4-62.5$ & \\
& Shift change & 62.5 & 1.7 & 57.7 & 67.8 & $61.9-63.0$ & 0.000 \\
& Midnight & 62.3 & 2,0 & 56.1 & 64.0 & $61.1-61.1$ & \\
& Morning & 64.7 & 2.3 & 59.8 & 69.6 & $63.9-65.4$ & \\
& Ward round & 64.5 & 2.1 & 59.7 & 70.0 & $63.8-65.1$ & \\
& Lunchtime & 63.9 & 2.0 & 61.2 & 70.5 & $64.0-65.3$ & \\
& Shift change & 65.0 & 2.5 & 61.4 & 72.5 & $64.1-65.7$ & \\
& Midnight & 63.2 & 2.0 & 58.0 & 66.3 & $64.0-64.7$ & 0.003 \\
& Morning & 66.4 & 2.5 & 59.3 & 72.6 & $65.6-67.2$ & \\
& Ward round & 67.1 & 2.4 & 63.3 & 76.5 & $66.3-67.8$ & \\
& Lunchtime & 65.9 & 1.9 & 61.5 & 71.0 & $65.3-66.5$ & \multirow{2}{*}{0.001} \\
& Shift change & 66.5 & 2.2 & 63.1 & 74.0 & $65.8-67.2$ & \\
& Midnight & 65.1 & 1.9 & 61.8 & 70.2 & $64.5-65.7$ & \\
& Morning & 65.3 & 4.0 & 55.0 & 74.8 & $64.9-66.5$ & \\
& Ward round & 65.3 & 4.2 & 55.0 & 75.0 & $64.0-66.6$ & \\
& Lunchtime & 62.2 & 4.1 & 50.3 & 68.0 & $61.0-63.5$ & \multirow{2}{*}{0.001} \\
& Shift change & 63.9 & 5.3 & 54.1 & 77.0 & $62.2-65.5$ & \\
& Midnight & 68.3 & 1.8 & 64.2 & 72.0 & $67.7-68.8$ & \\
& & & & & & & \\
& & & & &
\end{tabular}

SD: standard deviation Min: minimum Max: maximum CI: Confidence interval

Table 3 shows a summary of all hospitals during different shifts of the day. Lunchtime and midnight had the least noise level compared to other shifts. The noise level was high during ward rounds in all hospitals. It was also high during shift changing in hospitals 2 and 3 but less in hospitals 1 and 4 . Hospital 1 had the highest noise level during ward round $(63.9 \mathrm{~dB})$ and night duty shift $(63.2 \mathrm{~dB})$ 
compared to other shifts. The results of hospital 1 during different shifts showed statistically significant differences $(p<0.001)$. The second hospital had the largest noise during shift changing with a level of $65 \mathrm{~dB}$ followed by morning time. All this time, there is a handover of neonates to the replacing shift. The result was statistically significant $(p=0.003)$ at this hospital. Hospital 3 had the highest noise during ward round $(67.1 \mathrm{~dB})$ and shift changing $(66.5 \mathrm{~dB})$. The result of this hospital was statistically significant $(\mathrm{P}=0.001)$. Hospital 4 had the highest noise during the night shift $(68.3 \mathrm{~dB})$, followed by ward round $(65.3 \mathrm{~dB})$, and the result was statically significant $(P=0.001)$. The standard deviation was varied from one hospital to the other and had a larger variation in the fourth hospital.

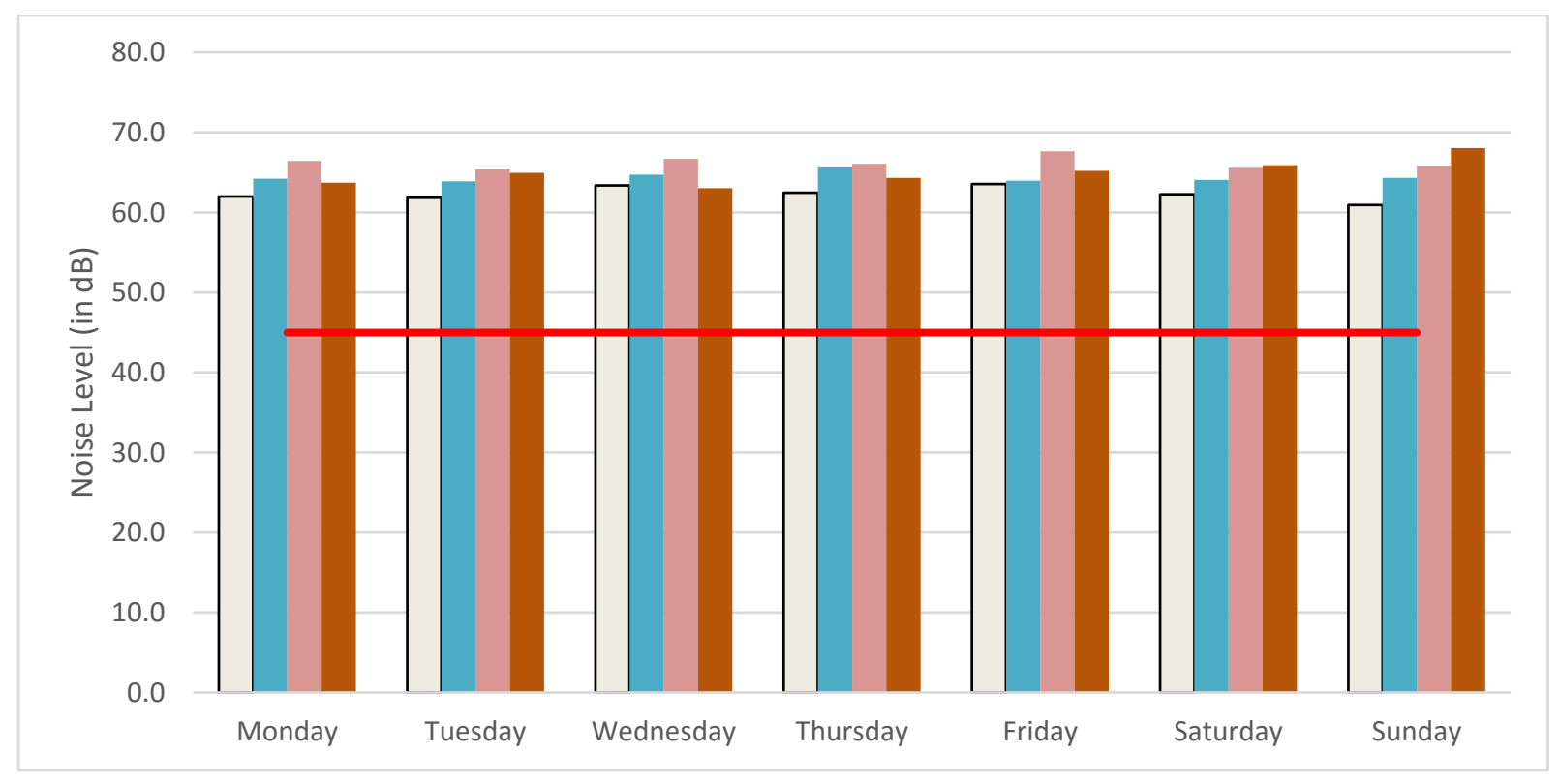

Figure 5. Comparing sound levels of NICU to the recommendation

WHO recommends that noise in hospital facilities should not exceed the mean sound of $35 \mathrm{~dB}$, and AAP recommended a mean sound of $45 \mathrm{~dB}$ in NICU (Schokry, 2016) (Figure 5). AAP was used as it is specific to NICU, whereas WHO is global to the whole hospital. Sound levels during a different day at different hospitals were above the recommendation of AAP, which is $45 \mathrm{~dB}$. It was statistical significant $(\mathrm{p}<0.05)$.

\section{DISCUSSION}

The average sound levels measured in the NICU of all four hospitals were above the maximum level of $45 \mathrm{~dB}$ recommended by the AAP in 1997. The basal noise level recorded in this study exceeds the noise level recommended by the WHO of $30-40 \mathrm{~dB}$ [14] and AAP of $45 \mathrm{~dB}$.[15] According to AAP, lack of compliance with the standard would result in the risk of adverse effects on the health of premature neonates.[15] High noise levels in NICU are a significant source of environmental stress to premature neonates, which affect; heart rate, respiratory rate, blood pressure, oxygen saturation, and intracranial pressure.[16] This sound level, which is higher than the recommended standards, has been noted in several studies carried out in various NICUs of Africa as well as internationally.[2]

\section{Measurement of noise level}

Sound levels varied based on time of day and location within the unit. The measured values were higher than the standard levels of the NICU, similar to other studies. A study done in South Australia reported; the maximum sound level was $74.5 \mathrm{~dB}$ in NICU.[17] Another study done in Portuguese NICUs showed a maximum sound level of $71.7 \mathrm{~dB}$, and in this study, the maximum sound to all hospitals in NICU was 77.0 dB.[6] The records of mean, maximum, and minimum sound levels were high and mainly during morning shifts compared to the night shift. The reason for high noise during the morning was due to more activities; such as faculty residents, undergraduate, nursing students round, administrative activities, in addition to noise generated by instruments and equipment.[18] 
The sound level during different shifts was high, > $45 \mathrm{~dB}$ and the difference was statistically significant, $p<0.05$. Noise levels increased during morning shifts and were higher at all hospitals compared to the night shift, similar to other studies.[17,19] The reduction of noise level during the night maybe was due to a small number of nurses at that time compared to the day shift. Sound levels during every shift of the day at all hospitals were statistically significant with $\mathrm{p}<0.05$, and also Blourchian, and Sharafi found out the noise level during each shift was statistically significant with $p=0.002$.[1] The sound was high on Thursday $65.6 \mathrm{~dB}$ at the hospital, and also a study done in Portugal found out that Thursday sound level was high at $79.7 \mathrm{~dB}$.[6] Noise level was low on Tuesday at hospital two, and another study showed that it was lowest at 53.6 dB on Saturday.[6]

The highest measure among the day time was during ward rounds and shift-changing in all hospitals. Ward rounds were the noisiest times due to the highest number of people present in the round. In a study done in Iran, it was found that sound levels had a direct relationship with the number of people present at the ward $(\mathrm{p}=$ 0.007).[20] While shift changing is the time nurses do handover leading to increased noise. Ward round mean sound levels in all four hospitals $(1,2,3$ and 4) were $64 \mathrm{~dB}, 64.5 \mathrm{~dB}, 67.1 \mathrm{~dB}$, and $65.9 \mathrm{~dB}$ respectively, which was consistent with other studies done by Ramm [17] with $52.4 \mathrm{~dB}$ and Joshi and Tada, which was between $50-95 \mathrm{~dB}$.[18] The lowest sound level among day shifts was seen during the midnight shift within three hospitals (1, 2 , and 3), $62.3 \mathrm{~dB}, 63.2 \mathrm{~dB}$, and $65.1 \mathrm{~dB}$, respectively. Another study detected the lowest measure at night with $61 \mathrm{~dB}$.[20]

The result for station/location one, which is near the entrance at each hospital (hospital 1, 2, 3, and 4), was high compared to other stations with measures $63.7 \mathrm{~dB}, 64.8 \mathrm{~dB}, 67.1 \mathrm{~dB}$ and $65.5 \mathrm{~dB}$ respectively. This is the same as the study done by Neille et al. ,[5] that reported that the sound level was higher on the measurement taken near the entrance $(67,6 \mathrm{~dB})$ and near nurses tea room $(62.4$ $\mathrm{dB})$. The sound level was also high near the nurses' station, which was ranging from $62.4 \mathrm{~dB}$ to $66.8 \mathrm{~dB}$. Sound level, as reported by Valizadeh during 6 locations, showed non-significant differences, $\mathrm{p}=$ 0.135 between sound levels, this is the same as hospitals 2 and 4, which was not statistically significant, $p=0.7$ and $p=0.9$, respectively.[20]

\section{Comparing noise level with recommendation}

The mean sound in this study was varying between 61.8 - $77.0 \mathrm{~dB}$, which was higher than the recommended level of AAP. A study done by Schokry showed a variation between 56 and $81 \mathrm{~dB}$, which was higher than the level recommended by AAP.[10] This was the same as the study done in Santa Marta- Colombia [19] in their study with sound ranging from $49-92 \mathrm{~dB}$, which was above the recommendation. A study done in Iran reported that sound levels ranged between $56.10 \mathrm{~dB}$ and 104.80 $\mathrm{dB}$, which were higher than standard levels according to the recommendation of AAP. [20] Another study done in an urban city similar to this study with an increased number of neonates reported the mean sound level ranging 51 - $95 \mathrm{~dB}$, higher than the recommended level.[18]

A study done in Portugal showed that noise levels were excessive in all the areas of the NICUs, exceeding the recommendation, with levels ranging between $48.7 \mathrm{~dB}$ to $71.7 \mathrm{~dB}$.[18] The average level of noise was $11-14 \mathrm{~dB}$ above the recommendation of AAP.[2] An exceptional study reported that; the lowest A-weighted sound level of $38 \mathrm{~dB}$ was reported only from one nursery in Lund, Sweden, which was the only nursery that concurred with the AAP recommendations for sound levels in NICU to be below $45 \mathrm{~dB}$.[11] In the study by Parra, the sound level was higher than the recommended by AAP ranging from $65.4 \mathrm{~dB}$ to $97 \mathrm{~dB} .[21]$

\section{Sources of noise level}

The findings from this study revealed that; the high noise level was due to numerous sources of the sound. The possible sources may be the following; talking/conversations, staff activity, monitors, alarms, phones, and others. Another study done in South Africa reported the same that conversations were always higher compared to other sources of noise, and another largest sound source was alarm monitors in a study done in Iraq.[22] Another study found out that phone ringing tones and neonatal crying had the highest result.[1] This was similar to what was observed with the phone ringing, and the neonate was crying in all hospitals. Noise sources were greater than $45 \mathrm{~dB}$, with the exceptional highfrequency oscillatory noise from the ventilator,[5] which was absent in all NICU during the time of data collection.

Only one hospital among the four has a ventilator, but during data collection, it was not administered to any neonate. So there was no source of ventilator noise at any of the hospitals. Some of the sources of noise can be prevented, e.g., HCP conversation, 
phone ring, etc., but others like alarm monitors, infusion pumps. Also, a study done by Santos suggested that this equipment could be modified for the safety of the neonates.[6] HCP and family members' conversation, the dropping equipment, contribute to the high sound level in NICU.[23]

\section{Limitations}

There are no appropriate specific standard limits for NICU; the one in use that was a bit specific to neonates was AAP, but it is specific as compared to WHO. There was no NICU standard sound level in the national guidelines. The readings were not taken inside the incubator but taken close to the neonate's head in an attempt to record a representative sound level reading. However, this might not have shown the exact noise level that affects the newborn. Noise sources were not measured in order to elaborate on the level of noise they contributed. There may still have been bias due to "halo effect", as HCP may have pretended and changed the behaviour, though precautions were taken to average noise levels and keep bias to a minimum.

\section{Recommendations}

To the Ministry of Health we recommend to establish national standard limits of sound level specifically for NICU in the national guidelines. Hospitals have to avoid unreasonable noise that seems to be necessary, such as conversations, which were seen at all hospitals. They have to use pipeline oxygen; that is fixed through the wall in order to decrease sound level exposure to neonates. Future research should measure the level of the noise source. The sound level should also be measured in rural areas and other hospitals where the research was not conducted.

\section{CONCLUSION}

In all the NICUs, the noise level generated was higher than the safe limits established by the WHO and AAP. Noise levels were highest during the ward rounds and at the station near the entrance in all hospitals. The noise level ranged from $61.8 \mathrm{~dB}$ to 77.0 dB, which was above the AAP safe recommendation and therefore affected neonatal care. The most frequent noise source in the NICU was HCP conversation, which is modifiable. This strategy will play a big part in noise reduction and monitoring in order to prevent the adverse effects of high noise levels on vulnerable neonates. Advocacy for a noise-free environment in NICUs is vitally needed for improved health of neonates in Rwanda.

\section{Acknowledgments}

We acknowledge all participants that made this study successful and Professor Oluyinka Adejumo for his great support and input in this study.

This article is published open access under the Creative Commons Attribution-NonCommercial NoDerivatives (CC BYNCND4.0). People can copy and redistribute the article only for noncommercial purposes and as long as they give appropriate credit to the authors. They cannot distribute any modified material obtained by remixing, transforming or building upon this article. See https://creativecommons.org/licenses/ by-nc-nd/4.0/

\section{REFERENCES}

1. Jahangir Blourchian M, Sharafi R. Determination of noise level and its sources in the neonatal intensive care unit and neonatal ward. Iranian Journal of Neonatology IJN. 2015 Dec 1;6(4):21-4.

2. Smith SW, Ortmann AJ, Clark WW. Noise in the neonatal intensive care unit: a new approach to examining acoustic events. Noise \& Health. 2018 Jul;20(95):121

3. Jordão MM, Costa R, Santos SV, Orlandi M, Locks H, Assuiti FC, et al. Noise in the neonatal unit: identifying the problem and proposing solutions * Cogitare Enferm. $2017 ;(22)$.

4. Aly HA, Ahmed AM. Effect of noise on neonatal vital data and dehavior in NICU. Clin Med Diagnostics. 2016;6(1):1-6.

5. Neille J, George K, Speech BA, Th H. A study investigating sound sources and noise levels in neonatal intensive care units. S Afr $\mathrm{J} \mathrm{CH}$. 2014;8(1):6-10.

6. Santos J, Carvalhais C, Xavier A, Silva MV. Assessment and characterization of sound pressure levels in Portuguese neonatal intensive care units. Archives of Environmental \& Occupational Health. 2018 Mar 4;73(2):121-7.

7. WHO, 2017 retrieved from http://www.who.int/mediacentre/factsheets/fs363/en/ accessed on 1/5/2018

8. US Agency for International Development, Project Concern International, Rwanda: Profile of preterm and low birth weight prevention and care, 2015 available at https://reliefweb.int/report/rwanda/rwanda-profile- 
preterm-and-low-birth-weight-preventionand-care accessed on 15th/4/2018

9. Neumann K, Chadha S, Tavartkiladze G, Bu $X$, White KR. Newborn and infant hearing screening facing globally growing numbers of people suffering from disabling hearing loss. International Journal of Neonatal Screening. 2019 Mar;5(1):7.

10. Schokry A. Study of noise levels in neonatal intensive care units (NICU) in public hospitals in Gaza City, Gaza Strip. Int J Eng Innov Technol. 2016;6(3):1-7.

11. D'Souza SR, Lewis LE, Kumar V, Kamath A, Nayak BS, Noronha JA, George A. Noise in neonatal intensive care unit: effects on hospitalized preterm infants. Manipal Journal of Nursing and Health Sciences (MJNHS). 2015; 1(1):57-62.

12. Cardoso SM, Kozlowski LD, Lacerda AB, Marques JM, Ribas A. Newborn physiological responses to noise in the neonatal unit. Brazilian Journal of Otorhinolaryngology. 2015 Dec;81(6):583-8.

13. Almadhoob A, Ohlsson A. Noise reduction management in the neonatal intensive care unit for preterm or very low birthweight infants ( Protocol ). Cochrane Database Syst Rev. 2015;1(1).

14. Berglund B, Lindvall T, Schwela DH. New WHO guidelines for community noise. Noise \& Vibration Worldwide. 2000 Apr;31(4):249.

15. American Academy of Pediatrics. Noise: a hazard for the fetus and newborn (RE9728). Pediatrics. 1997 Oct;100(4):1-0.

16. Peng NH, Bachman J, Jenkins R, Chen CH, Chang YC, Chang YS, Wang TM. Relationships between environmental stressors and stress biobehavioral responses of preterm infants in NICU. The Journal of Perinatal \& Neonatal Nursing. 2009 Oct 1;23(4):363-71.

17. Ramm K, Mannix T, Parry Y, Gaffney MP. A comparison of sound levels in open plan versus pods in a neonatal intensive care unit. HERD: Health Environments Research \& Design Journal. 2017 Apr;10(3):30-9.

18. Joshi G, Tada N. Analysis of noise level in neonatal intensive care unit and post-natal ward of a tertiary care hospital in an urban city. International Journal of Contemporary Pediatrics. 2016;4(3):1358-61.

19. Garrido Galindo AP, Camargo Caicedo Y, Velez-Pereira AM. Noise level in a neonatal intensive care unit in Santa Marta-Colombia. Colombia Médica. 2017 Sep;48(3):120-5.

20. Valizadeh S, Hosseini MB, Alavi N, Asadollahi M, Kashefimehr S. Assessment of sound levels in a neonatal intensive care unit in Tabriz, Iran. Journal of Caring Sciences. 2013 Mar;2(1):19.

21. Parra J, de Suremain A, Berne Audeoud F, Ego A, Debillon T. Sound levels in a neonatal intensive care unit significantly exceeded recommendations, especially inside incubators. Acta Paediatrica. 2017 Dec;106(12):1909-14.

22. Aljawadi HF, Ali EA, Mahmood MH. Noise level at neonatal care unit of Al-Sader Teaching Hospital in Misan Province, Iraq. Kerbala Journal of Medicine. 2017;10(2):2740-6.

23. Sá MM, Azevedo R, Neves J, Machado O, Tavares J. Noise in an intensive care nursery/newborn unit. J Health Educ Res Dev. 2018;6(265):2. 